\title{
Effects of potential calcium sensing receptor inducers on promoting chemosensitivity of human colon carcinoma cells
}

\author{
XIZE WANG $^{1}$, WEI CHEN ${ }^{1}$, NAVNEET SINGH ${ }^{2}$, MULTIRA PROMKAN $^{2}$ and GUANGMING LIU ${ }^{2}$ \\ ${ }^{1}$ Department of General Surgery and Department of Experimental Medicine, Liaoning Northern Hospital, \\ Shenyang 110016, Liaoning, P.R. China; ${ }^{2}$ SimmonsCooper Cancer Institute, Southern Illinois \\ University School of Medicine, Springfield, IL 62702, USA
}

Received January 18, 2010; Accepted February 26, 2010

DOI: 10.3892/ijo_00000644

\begin{abstract}
We have previously reported that by inducing calcium sensing receptor (CaSR), calcitriol, the active form of vitamin $\mathrm{D}$, promoted the sensitivity of the human colon carcinoma cells to anticancer drugs. In the current study we tested several other potential CaSR modulators, calcipotriol and the effective components of Chinese herbal medicine lentinan, for their functions in inducing CaSR expression in human colon carcinoma cell line CBS, Moser, Fet, and SW480 cells and subsequently promoting sensitivity of the cells to anticancer drugs. Calcipotriol and lentinan suppressed invasion of the colon carcinoma cells and enhanced the cytotoxicity of anticancer regimen FOLFIRI to cells in culture or in anchorage-independent growth. In the mechanism study we found that calcipotriol and lentinan suppressed protein expression and gene transcriptional activities of survivin and thymidylate synthase, increased E-cadherin cell membrane localization and complex formation of E-cadherin and Bcatenin, and repressed TCF4 transcriptional activation. These effects were attenuated, however, in CaSR knocked-down cells, indicating that CaSR was required in the pathway. We concluded that calcipotriol and lentinan are efficient in promoting chemosensitivity in colon carcinoma cells. Since both compounds have much less side effects than calcitriol in clinic, they have greater potential to be applied as supplements of colon cancer therapy.
\end{abstract}

Correspondence to: Dr Guangming Liu, SimmonsCooper Cancer Institute, Southern Illinois University School of Medicine, Springfield, IL 62794-9677, USA

E-mail: gliu@siumed.edu

Abbreviations: CaSR, calcium sensing receptor; TS, thymidylate synthase; FOLFIRI, 5-FU, leucovorin, and SN-38; shRNA-CaSR, shRNA expression vector targeting the CaSR; shRNA-Ctrl, shRNAscrambled control vector

Key words: calcipotriol, lentinan, calcium sensing receptor, human colon carcinoma cells, drug sensitivity

\section{Introduction}

CaSR is a $\mathrm{G}$ protein-coupled receptor originally identified in human parathyroid gland where it senses the instant changes in extracellular $\mathrm{Ca}^{2+}$ levels (1). It was discovered later that CaSR is also expressed in a wide variety of human tissues, including human colon cancer and other cancer cells (2-4). The potential function of CaSR in colon epithelium is involved in promoting the progress of cell differentiation. In normal colon epithelium, there is no CaSR expression at the bottom of the colonic crypts where undifferentiated stem cells are located and responsible for epithelial tissue renewal $(5,6)$. As the epithelial cells differentiate and migrate upward to the top of the crypt, the cells acquire a gradient increase in the expression of the CaSR $(5,6)$. In colon cancer, welldifferentiated carcinoma cells express observable levels of CaSR, whereas undifferentiated carcinoma cells have little or no CaSR expression (7-10). Since undifferentiated status is one of the mechanisms underlying drug resistance, we have hypothesized in our previous studies that CaSR is involved in chemosensitivity of human colon carcinoma cells and found that activation or induction of CaSR in the cells significantly promoted the sensitivity of the cells to anticancer drug treatment $(11,12)$.

The human CaSR gene has two promoters (P1 and P2) and each promoter has a transcriptional start site (4,13-15). Both promoters contain the vitamin D (VD) response element (VDRE), which is recognized and bound by the complex of VD receptor (VDR) activated and bound by VD (15). Through this mechanism, VD induces $\mathrm{P} 1$ and $\mathrm{P} 2$ promoter transcriptional activity (7), up-regulates CaSR mRNA levels, and increase CaSR protein expression (7). We have previously reported that calcitriol, the active form of $\mathrm{VD}$, induced the expression of CaSR in human colon carcinoma cells, which led to an increase in the sensitivity of the cells to anticancer drug treatment. This observation is consistent with the well-known function of VD for its anticancer effects in colon (17-19). VD, however, can not be used in clinic as an anticancer drug supplement due to its hypercalcemic side effect. Calcipotriol (1- $\alpha$-hydroxyvitamin D3) is a synthetic derivative of calcitriol used in the treatment of psoriasis. This drug has an excellent safety profile with rarely reported hypercalcemic side-effect (20). If calcipotriol retains the same function as calcitriol in 
terms of inducing CaSR in colon carcinoma cells, it will be an ideal candidate for the potential use as a supplemental agent for anticancer therapy in colon. Such effect of calcipotriol, however, has never been tested. Lentinan is the Shiitake Mushroom polysaccharides, which elicits anticancer functions through suppressing proliferation and promoting differentiation in colon carcinoma cells (21). It is unclear whether the CaSR is regulated by this compound as an underlying mechanism.

In the present study, we tested calcipotriol and lentinan for their functions in inducing CaSR expression in human colon carcinoma cell line CBS, Moser, Fet, and SW480 cells and subsequently promoting sensitivity of the cells to anticancer drugs. We found that calcipotriol and lentinan had strong effects in inducing CaSR and increasing chemosensitivity. In the mechanism study we found that calcipotriol and lentinan suppressed protein expression and gene transcriptional activities of survivin and thymidylate synthase (TS), increased E-cadherin cell membrane localization and complex formation of E-cadherin and B-catenin, and repressed TCF4 transcriptional activation in a CaSR-dependent manner. Since both compounds have much less side effects than calcitriol in clinic, they have greater potential to be used as supplements of colon cancer therapy.

\section{Materials and methods}

Cell culture and treatment. Human colon carcinoma cell line CBS, Moser, Fet, and SW480 cells were maintained in SMEM medium (Sigma, St. Louis, MO) as described previously (7-9). To assess the effect of the compounds indicated on the cytotoxic response to anticancer drugs, the medium of actively growing cells was replenished with medium containing the compounds at the doses indicated in the figures and FOLFIRI (5-FU $60 \mu \mathrm{M}$, leucovorin $28 \mu \mathrm{M}, \mathrm{SN}-3840 \mu \mathrm{M}$ ). The medium of a parallel set of cultures was replenished with medium only containing FOLFIRI without the addition of exogenous reagents. The medium of control cultures was replenished with regular culture medium without FOLFIRI or exogenous reagents. Cells were then incubated at $37^{\circ} \mathrm{C}$ in a $\mathrm{CO}_{2}$ incubator for the time periods as indicated in the figure legends. 5-FU, leucovorin, SN-38, calcipotriol were purchased from Sigma. Calcitriol was purchased from Cayman Chemical (Ann Arbor, MI). Lentinan was purchased from Hangzhou Kangbaier Bio-Technology Co., Ltd. (Hangzhou, China).

Cell transfection. shRNA-CaSR plasmid was constructed as previously described (11). Plasmids of shRNA-CaSR or shRNA-scrambled control were transfected into CBS, Moser cells and the stable shRNA-CaSR or shRNA-scrambled transfectants were established by selection and maintenance of the cells with antibiotics as previously described (11).

Determination of cell viability. The number of viable cells was determined using Vi-Cell ${ }^{\mathrm{TM}}$ XR Cell Viability Analyzer (Beckman Coulter, Inc., Fullerton, CA). In brief, colon carcinoma cells and their shRNA-CaSR transfectants were treated with FOLFIRI at the absence or presence of calcitriol, calcipotriol or Lentinan as indicated in the figures and figure legends. The cells were then trypsinized and resuspended in PBS buffer. Viable cells were counted using the instrument.
Cell lysis preparation and Western blot analysis. Western blottings were performed essentially as previously described $(7,9)$. Cells were treated as indicated and then total cell lysates and membrane proteins were prepared. For total cell lysates, the cells were lysed in lysis buffer. The cell membrane lysates were prepared using The Mem-PER Mammalian Membrane Protein Extraction Reagent Kit (Thermo Scientific, Rockford, IL) according to the manufacturer's instructions. The protein concentration was determined using the Bio-Rad assay system (Bio-Rad, Hercules, CA). Anti-human CaSR antibody was purchased from Millipore (Billerica, MA). Anti-human TS antibody was purchased from Zymed Laboratories, Inc. (Carlsbad, CA). Anti-human survivin antibody was purchased from R\&D Systems, Inc. (Minneapolis, MN). Anti-human E-cadherin and anti-human $\beta$-catenin antibody were purchased from Abcam Inc. (Cambridge, MA). Horseradish peroxidaseconjugated anti-rabbit, anti-mouse, or anti-goat $\operatorname{IgG}$ was used as the secondary antibody and the protein bands were detected using the Fujifilm LAS-3000 system (Fujifilm Life Science, Stamford, CT). The plasma membrane marker $\alpha 2$-integrin was used as a control for equal extraction of cell membrane protein. B-actin was used as internal controls to evaluate the uniformity of total cell lysates protein loading. Antibodies against $\alpha 2$-integrin or $\beta$-actin were purchased from Abcam Inc.

Immunoprecipitations. Cell lysates containing $200 \mu \mathrm{g}$ membrane protein were immunoprecipitated by $3 \mu \mathrm{g}$ of monoclonal antibody against E-cadherin. The complex was then pulled down by Sepharose-conjugated protein $\mathrm{G}$ beads (Thermo Fisher Scientific, Rockford, IL.) at $4^{\circ} \mathrm{C}$ with gentle tumbling overnight. Immunoprecipitates were washed 4 times, eluted, and then analyzed by Western blotting with antibodies against E-cadherin and ß-catenin.

Luciferase reporter assays. The cell lines and their shRNACaSR stable transfectants were seeded in 24-well plates and grown to $70-80 \%$ confluence. Cells were transfected with $0.7 \mu \mathrm{g}$ of either survivin promoter luciferase reporter plasmids pLuc-2840 or TS promoter luciferase reporter plasmids 3RG and $0.1 \mu \mathrm{g}$ of Renilla luciferase reporter control plasmid per well, using Lipofectamine ${ }^{\mathrm{TM}} 2000$ (Invitrogen Life Technologies, Frederick, MD), as previously described $(7,9,22)$. Chemicals indicated were added $24 \mathrm{~h}$ after transfections, and luciferase activities were measured at $24 \mathrm{~h}$ after treatment. Transfection efficiencies were determined by Renilla luciferase activities. Survivin promoter luciferase reporter plasmid pLuc-2840 was a gift from Dr F. Li (23). TS promoter luciferase reporter plasmid $3 R G$ was a gift from Dr R.D. Ladner (24). T cell factor (TCF) transcriptional activation activity was measured using the luciferase reporter pTOPFLASH/pFOPFLASH plasmids as previously reported (9) and performed as above described.

Invasion assay. The invasion assay was performed by using 24-well BD Biocoated Matrigel invasion chambers with $8-\mu \mathrm{m}$ polycarbonated filters (BD Biosciences, Bedford, MA), as described previously (25). In brief, the four cell lines and their shRNA-CaSR transfectants were seeded on Matrigel invasion chamber at $10^{5}$ cells per well. The cells were cultured in $5 \%$ FBS/SMEM medium in the absence or presence of the 
a

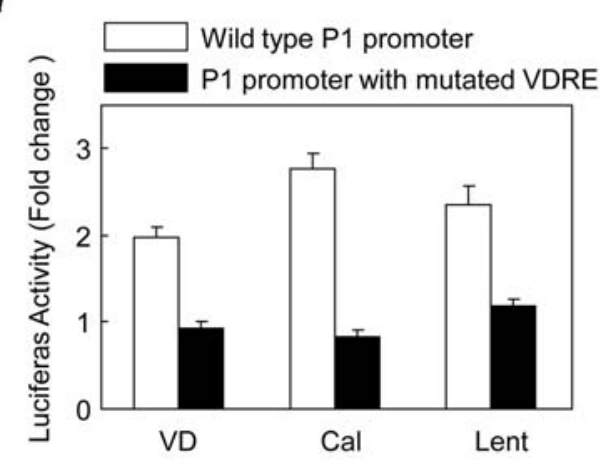

$b$
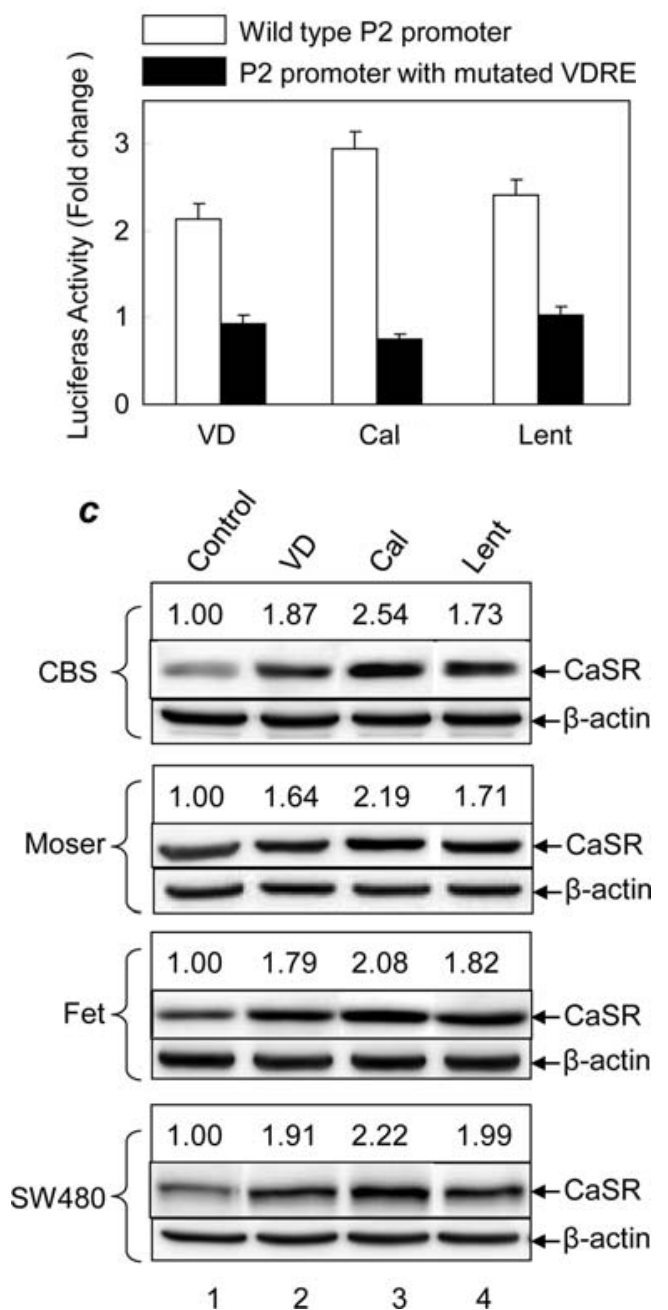

Figure 1. Expression of CaSR is inducible by the compounds tested in human colon carcinoma cells. (a) Human colon carcinoma CBS cells transfected with wild-type P1 promoter luciferase reporter plasmid or VDRE mutated P1 promoter luciferase reporter plasmid were treated with or without the compounds indicated for $24 \mathrm{~h}$ and then luciferase activities were determined. The data are represented as fold changes over that of cells without treatments. Empty bar, cells transfected with the wild-type P1 promoter luciferase reporter plasmid; solid bar, cells transfected with the VDRE mutated P1 promoter luciferase reporter plasmid. (b) CBS cells transfected with P2 promoter or VDRE mutated P2 promoter were treated and measured as described in (a). The error bars represent the standard error of the mean of triplicate experiments. (c) The cells indicated were treated with the chemicals for $24 \mathrm{~h}$ and CaSR expression was determined by Western blots as described in Materials and methods. Numbers on blots represent the levels of CaSR expression compared to untreated cells with an assigned value of 1 . Ctrl, cells without exposure to the chemicals as control; VD, calcitriol $(1 \mu \mathrm{M})$; Cal, calcipotriol $(1 \mu \mathrm{M})$; Lent, lentinan $(10 \mu \mathrm{M})$. chemicals indicated for $24 \mathrm{~h}$. Invasive cells that penetrated through Matrigel and migrated to the underside of the membrane were counted under microscopic vision after fixation with $4 \%$ formaldehyde in PBS. The average cell number of triplicate wells was determined.

Anchorage-independent growth assay. The soft agar assay testing the anchorage-independent growth in vitro was performed as previously described (26). In brief, $10^{4}$ cells of the four cell lines and their shRNA-CaSR transfectants were resuspended with $0.6 \mathrm{ml}$ of $0.3 \%$ agarose gel (Invitrogen Corp.) in $10 \%$ FBS/SMEM medium containing FOLFIRI at the absence or presence of the chemicals indicated. The cellagar mixture was immediately seeded into 24 -well plates coated with $0.6 \%$ agar in $10 \% \mathrm{FBS} / \mathrm{SMEM}$ medium at the absence or presence of the chemicals indicated. The cultures were maintained in a $37^{\circ} \mathrm{C}, 5 \% \mathrm{CO}_{2}$ incubator for 2-4 weeks, and the cell colonies were scored under microscopic vision. The average colony number of triplicate wells was determined.

\section{Results}

Calcipotriol and lentinan induce CaSR expression. We first screened the compounds for their effects on inducing CaSR promoter transcription activation by treating the cells transiently transfected with CaSR promoter P1 reporter plasmid or P1 promoter with mutated VDER and compared the result with calcitriol treatment. The result showed (Fig. 1a), that calcipotriol and lentinan induced a comparable P1 transcriptional activity as calcitriol did. When VDER site was mutated, however, the activation was abolished, indicating that the effects of these compounds were through VDER. Similarly, the compounds also induced a comparable transcriptional activity as calcitriol did in CaSR P2 promoter but not in P2 promoter with mutated VDRE (Fig. 1b), indicating that both $\mathrm{P} 1$ and $\mathrm{P} 2$ promoters are regulated through the VDRE sites. To further determine the CaSR protein expression induced by the compounds, we treated the cells with the compounds and performed Western immunoblot analysis to determine the CaSR levels. As shown in Fig. 1c, all the compounds tested induced a significant increase in CaSR expression.

Calcipotriol and lentinan promote chemosensitivity to FOLFIRI. We have recently reported that induction of CaSR in human colon carcinoma cells by calcitriol promoted the sensitivity of the cells to the anticancer drug 5-FU (12). Since calcipotriol and lentinan exhibited similar effects in inducing CaSR expression as calcitriol, we hypothesized that these compounds may also be able to strengthen chemosensitivity in human colon carcinoma cells. As shown in Fig. 2a, in the presence of either of the compounds, the human colon carcinoma cells killed by FOLFIRI were more than double as that killed by FOLFIRI alone. In the invasive assay, the invasive ability of the human colon carcinoma cells was significantly suppressed by the presence of either of the compounds (Fig. 2b). Clonogenic assays were further performed in soft agar to measure the cellular response to FOLFIRI in the absence or presence of the compounds. Fig. 2c shows that all the compounds increased the suppressive effect of FOLFIRI on colony formation. 
a
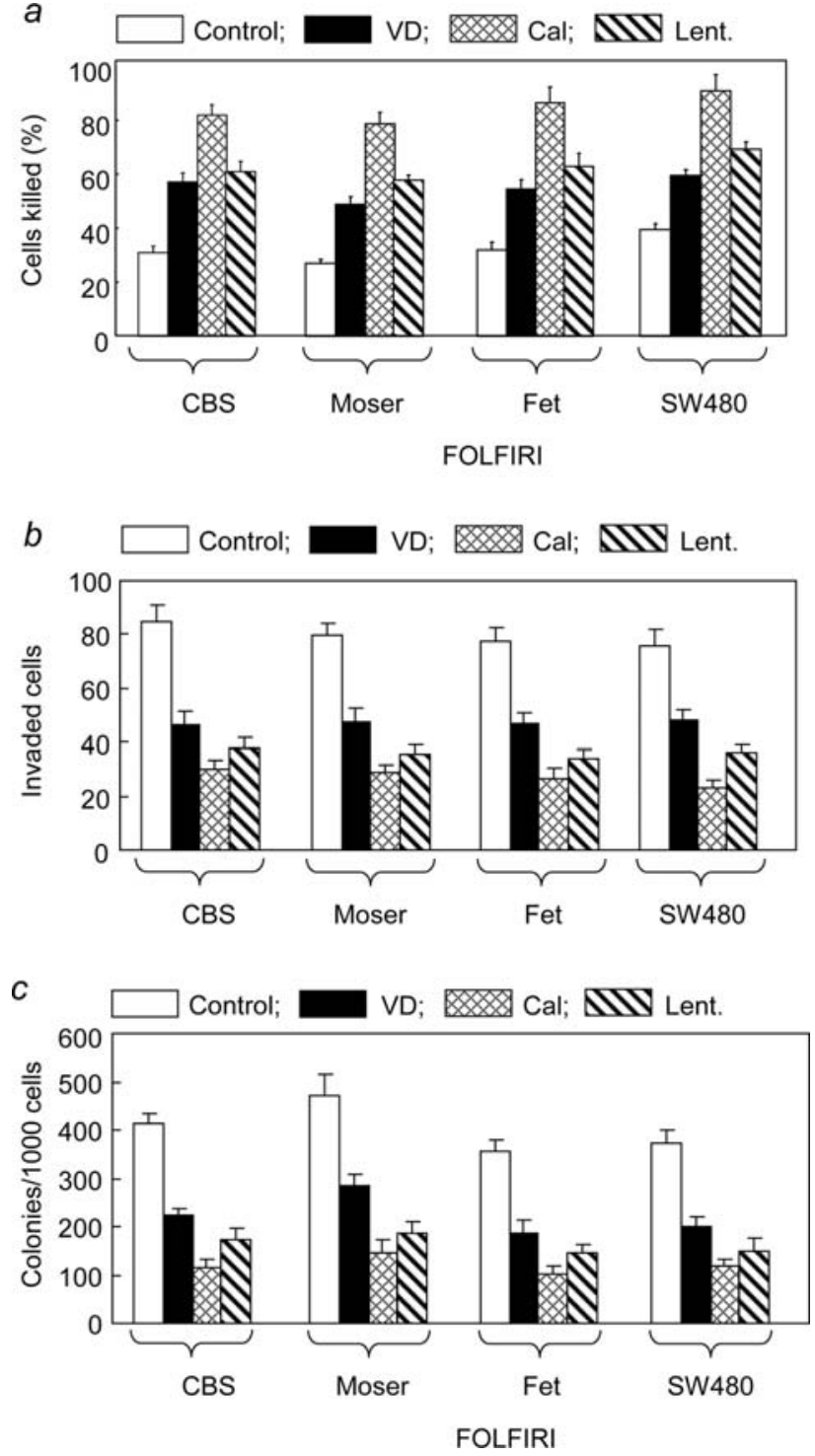

Figure 2. Calcipotriol and lentinan enhance FOLFIRI anticancer effects (a) The chemicals promote the sensitivity of the cells to FOLFIRI. Cells in 24-well plate were cultured in medium without or with FOLFIRI for $24 \mathrm{~h}$ Viable cells were counted as described in Materials and methods. Results are expressed as percent cells killed by comparison with cells cultured in the absence of FOLFIRI and the chemicals. (b) The chemicals inhibit cellular invasion. Cells were or were not exposed to the chemicals indicated for $24 \mathrm{~h}$ and the invasive assays were performed as described in Materials and methods using commercially available invasion chambers. (c) The chemicals enhance the suppressive effects of FOLFIRI on anchorage-independent growth of the human colon carcinoma cells. Clonogenic assays were performed as described in Materials and methods with cells growing on soft agar were exposed to FOLFIRI at the absence or presence of the chemicals indicated. The error bars represent the standard error of the mean of triplicate experiments. Control, cells without exposure to the chemicals; VD, calcitriol $(1 \mu \mathrm{M})$; Cal, calcipotriol $(1 \mu \mathrm{M})$; Lent, lentinan $(10 \mu \mathrm{M})$.

Calcipotriol and lentinan promote chemosensitivity in a CaSRdependent manner. As we have recently reported, CaSR is required in mediating calcitriol-promoted chemosencitivity in human colon carcinoma cells (12). We hypothesized that the same mechanism governed the effects of calcipotriol and lentinan. Using the CaSR knock-down cell model that we have previously prepared $(11,12)$ and maintained in good condition, as shown in Fig. 3a, we determined the effects of calcipotriol or
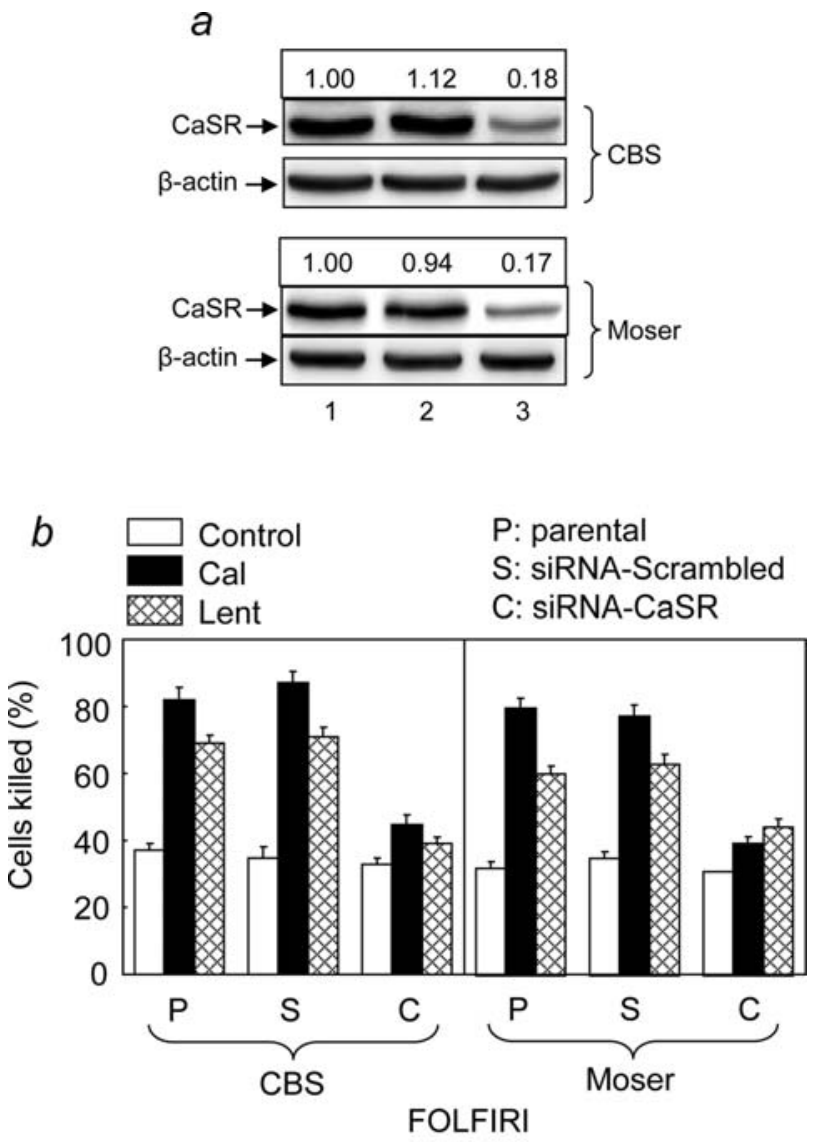

Figure 3. Calcipotriol and lentinan promote chemosensitivity in a CaSRdependent manner. (a) Transfection with shRNA-CaSR knocked down the expression of CaSR. Western blots of CaSR expression in shRNA-CaSRtransfected and control (shRNA-scrambled)-tranfected CBS and Moser cells. The expression of $B$-actin was used as internal control for equal protein loading. Lane 1, parental cells. Lane 2, shRNA-scrambled control transfected cells. Lane 3, shRNA-CaSR transfected cells. Numbers on blots represent the levels of CaSR expression by comparison to parental cells with an assigned value of 1. (b) Calcipotriol and lentinan enhance the cytotoxic response to FOLFIRI in a CaSR-dependent manner. CBS or Moser cells in 24-well plates were exposed to FOLFIRI for $24 \mathrm{~h}$ in medium without or with the chemicals. Viable cells were counted as described in Materials and methods. Results are expressed as percent cells killed by comparison with control cells cultured in the absence of FOLFIRI and the chemicals. The error bars represent the standard error of the mean of triplicate experiments. Control, cells without exposure to the chemicals; Cal, calcipotriol $(1 \mu \mathrm{M})$; Lent, lentinan $(10 \mu \mathrm{M})$; $\mathrm{P}$, parental cells; S, shRNA-scrambled transfected control cells; C, shRNACaSR transfected cells.

lentinan in the cell models. As shown in Fig. 3b, calcipotriol or lentinan promoted cytotoxic responses to FOLFIRI in CBS or Moser cells but not in their CaSR knocked down counterparts, indicating that the compounds promoted cell sensitivity to anticancer drugs in a CaSR-dependent manner.

Calcipotriol and lentinan modulate the expression of TS and survivin through CaSR. We further determined the potential mechanisms by which calcipotriol or lentinan modulates the cell sensitivity to FOLFIRI and whether CaSR function was required in the context. It is known that TS is the major cellular target of 5-FU (the major active component of FOLFIRI) and increased TS expression underlies 5-FU resistance (27) while increased expression of the anti-apoptotic protein survivin can confer resistance to a variety of anticancer 


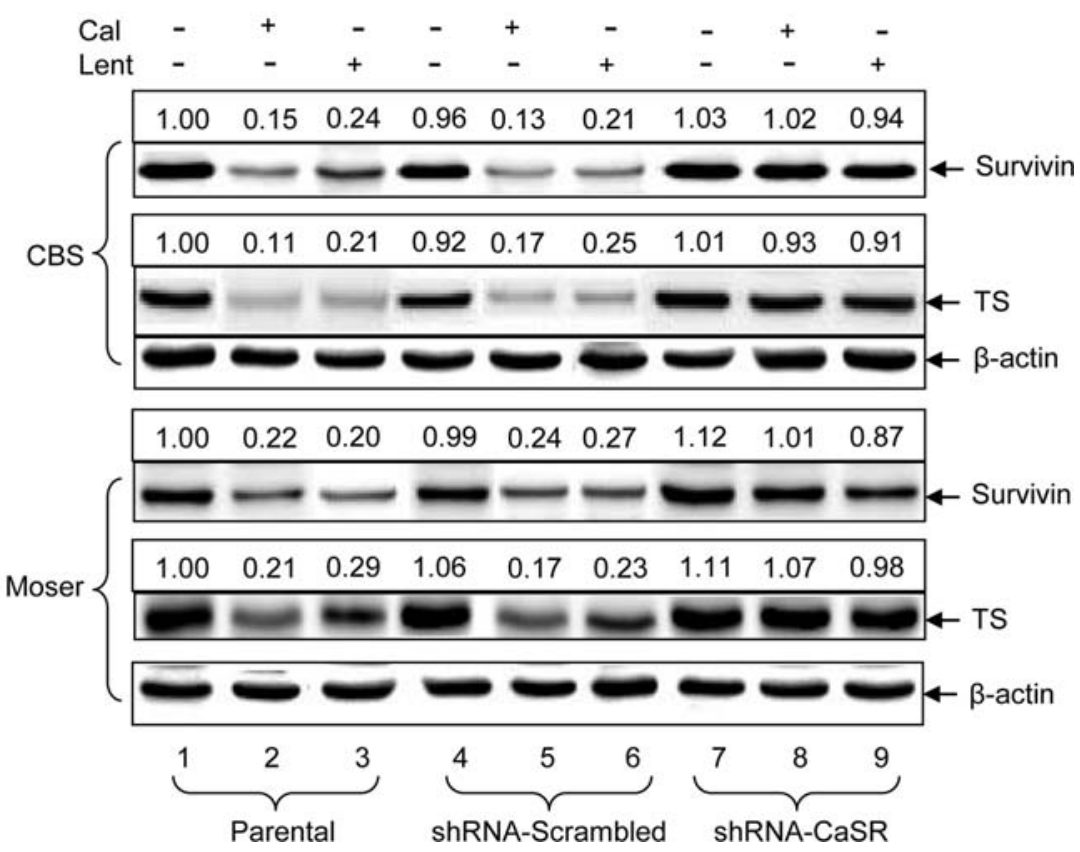

$b$

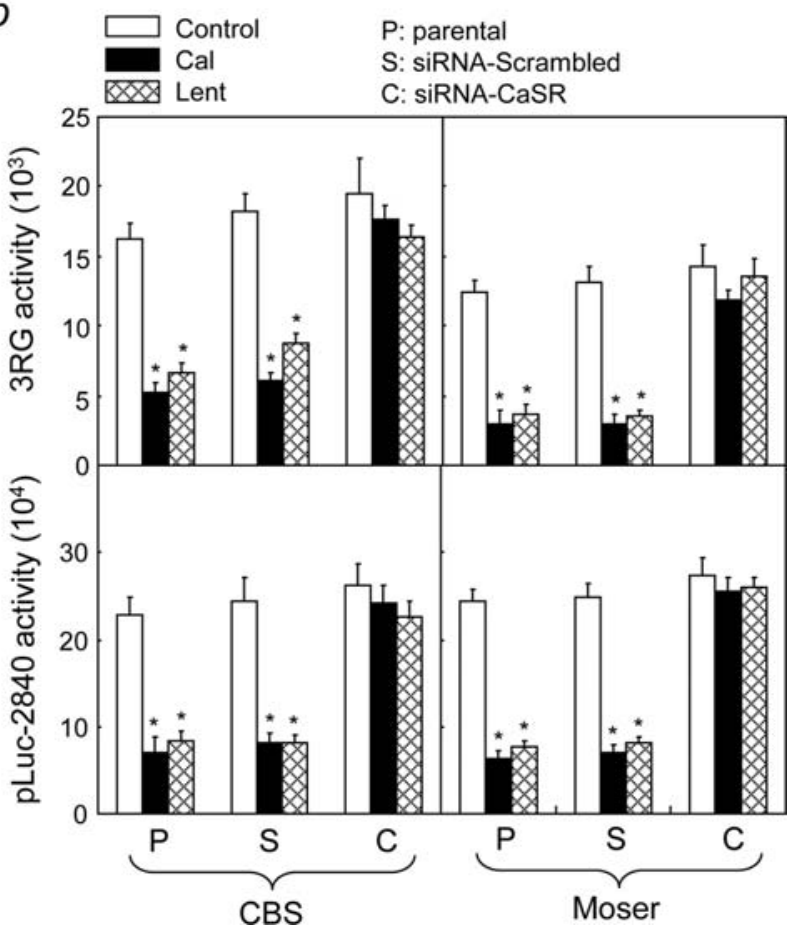

Figure 4. Calcipotriol and lentinan suppress the expression of TS or survivin in a CaSR-dependent manner. (a) CBS or Moser cells and their transfectants were treated without or with the chemicals indicated for $24 \mathrm{~h}$ and Western blot analyses for TS and survivin expression were performed as described in Materials and methods. B-actin was used as an internal control for equal protein loading. Numbers on top of each band indicate the levels of protein expression by comparison to controls (lane 1). (b) Calcipotriol and lentinan inhibit TS or survivin gene transcription in a CaSR-dependent manner. TS luciferase reporter 3RG plasmid and survivin luciferase reporter pLuc-2840 plasmid were prepared and the assays for TS or survivin gene transcriptional activities were performed as described in Materials and methods. Exposure of the human colon carcinoma cells to calcipotriol or lentinan for $24 \mathrm{~h}$ down-regulated TS (upper panel) or survivin (lower panel) promoter reporter luciferase activities. The error bars represent the standard error of the mean of triplicate experiments. Asterisk (*) indicates $\mathrm{p}<0.01$ compared with parental cells cultured in control medium. Control, cells without exposure to the chemicals; Cal, calcipotriol $(1 \mu \mathrm{M})$; Lent, lentinan $(10 \mu \mathrm{M})$; P, parental cells; $\mathrm{S}$, shRNA-scrambled transfected control cells; C, shRNA-CaSR transfected cells.

drugs $(28,29)$. If calcipotriol or lentinan could modulate cellular sensitivity to FOLFIRI, we hypothesized that TS and survivin might be the downstream events of CaSR modulated by these reagents. Our results showed that calcipotriol or lentinan downregulated the expression of TS and survivin in CBS or Moser parental cells or siRNA-scrambled control cells (Fig. 4a). Disrupting CaSR expression by shRNA-CaSR abrogated the modulating effect of the reagents on the expression of TS and survivin (Fig. 4a). Thus, like calcitriol, calcipotriol or lentinan functioned in down-regulation of TS and survivin through their modulating CaSR expression. Similar results were observed in gene transcriptional activities of survivin and TS. The cells were transfected with survivin promoter luciferase reporter plasmid pLuc-2840 (23) or TS promoter luciferase reporter plasmid 3RG (24) and luciferase reporter activities were assayed in these cells in response to treatment with extracellular calcipotriol or lentinan. As shown in Fig. 4b, extracellular calcipotriol or lentinan significantly suppressed pLuc-2840 transcriptional activity (Fig. 4b, upper panel) and 3 RG transcriptional activity (Fig. 4b, lower panel) in CBS or Moser cells but not in their shRNA-CaSR-transfected counterparts. These results show that calcipotriol or lentinan can suppress survivin and TS protein expression by downmodulating the transcription of survivin and TS genes and that CaSR is required to mediate this effect of calcitriol. When CaSR is no longer inducible in shRNA-CaSR tranfected cells, the effects of these compounds in this regard are abolished.

Calcipotriol and lentinan suppress $\beta$-catenin/TCF signaling. To determine the possible involvement of Wnt signaling in the effects of calcipotriol or lentinan through CaSR, we investigated the complex formation of E-cadherin/ß-catenin upon the treatment of the compounds. Our results showed that calcipotriol or lentinan significantly increased the expression of E-cadherin and B-catenin in cell membrane (Fig. 5a). They also increased the complex formation of E-cadherin and $B$-catenin in 


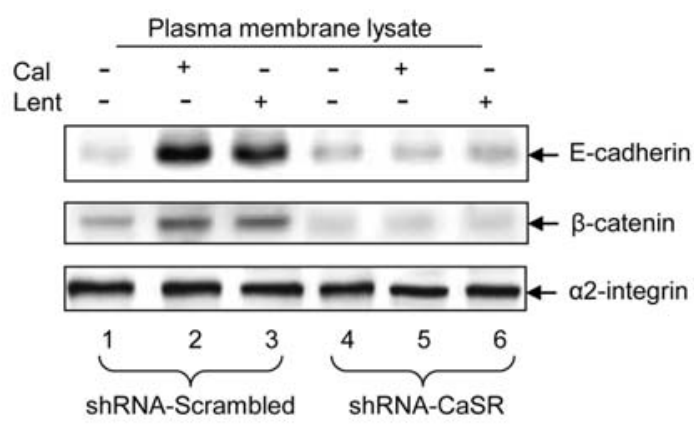

$b$
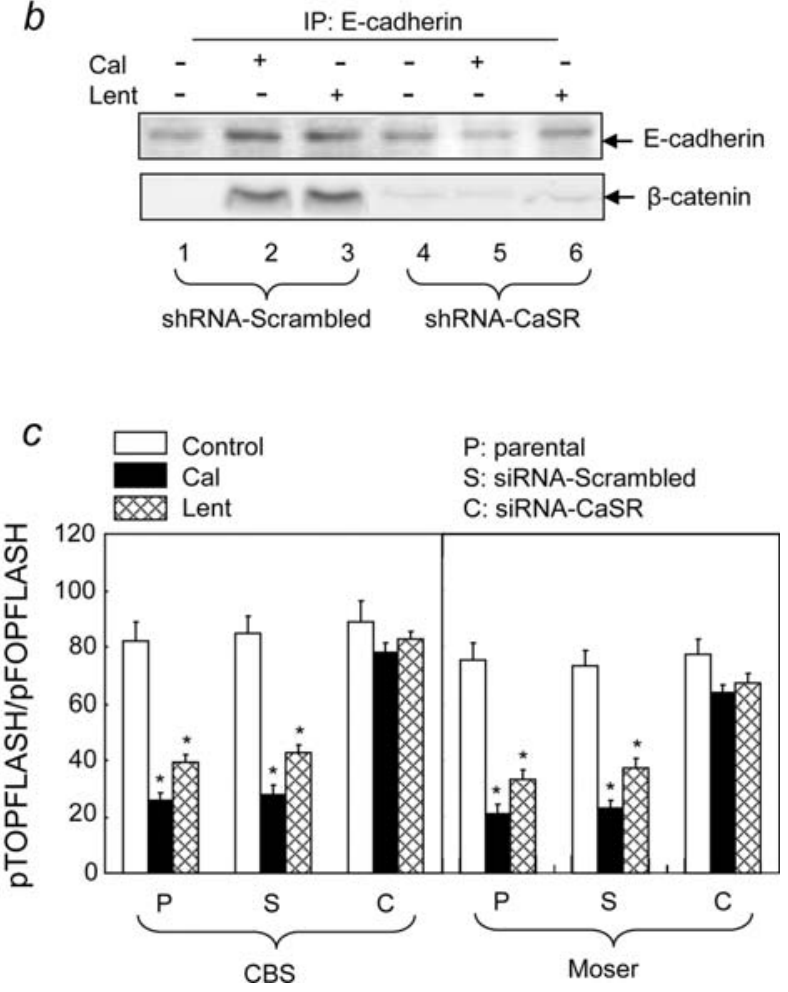

Figure 5. Calcipotriol and lentinan suppress $\beta$-catenin/TCF signaling pathway in a CaSR-dependent manner. (a) Calcipotriol and lentinan increase cell membrane localization of E-cadherin and B-catenin. CBS cells and their transfectants were treated without or with the chemicals indicated for $24 \mathrm{~h}$. The plasma membrane lysates were extracted and Western blot analyses for E-cadherin and B-catenin were performed as described in Materials and methods. (b) The plasma membrane lysates were immunoprecipitated (IP) with antibody against E-cadherin and the immunoprecipitates were then analyzed by Western blotting for E-cadherin and ß-catenin. $\alpha 2$-integrin (a plasma membrane marker) was used as a control for equal extraction of cell membrane proteins. (c) Calcipotriol and lentinan inhibit TCF4 gene transcription in a CaSR-dependent manner. TCF4 luciferase reporter pTOPFLASH and control pFOPFLASH plasmids were prepared and the assays of TCF4 gene transcriptional activities were performed as described in Materials and methods. Exposure of the human colon carcinoma cells to calcipotriol or lentinan for $24 \mathrm{~h}$ down-regulated TCF4 promoter reporter luciferase activities. The error bars represent the standard error of the mean of triplicate experiments. Asterisk (*) indicates $\mathrm{p}<0.01$ compared with parental cells cultured in control medium. Control, cells without exposure to the chemicals; Cal, calcipotriol $(1 \mu \mathrm{M})$; Lent, lentinan $(10 \mu \mathrm{M})$; P, parental cells; S, shRNA-scrambled transfected control cells; C, shRNA-CaSR transfected cells.

the plasma membrane (Fig. 5b). These effects, however, were abrogated in the CaSR knocked-down cells (Fig. 5a and b).
Accordingly, calcipotriol or lentinan suppressed TCF4 transcriptional activation activity represented by the luciferase reporter pTOPFLASH/pFOPFLASH activities in parental CBS cells but not in CaSR knocked-down cells (Fig. 5c). These results suggested that CaSR plays a key role in regulating Wnt signaling by increasing E-cadherin interaction with $\beta$-catenin in cytoplasmic membrane and thus preventing $\beta$-catenin translocation to nucleus where it forms $\beta$-catenin/TFC4 complex to drive the malignant progression of colon cancer.

\section{Discussion}

In this study, we investigated the effect of several potential CaSR inducers to enhance human colon carcinoma cell sensitivity to anticancer regimen FOLFIRI and the underlying mechanisms. We concluded that the vitamin $\mathrm{D}$ derivative calcipotriol and the Shiitake mushroom polysaccharides lentinan have great potential in inducing CaSR expression in human colon carcinoma cells, which was the key event for strengthening the cell response to anticancer drugs. This finding is interesting since calcipotriol and lentinan have much less side effects than calcitriol to human body. Therefore, they have greater potential to be applied as the supplements of colon cancer therapy.

A major problem in the treatment of colorectal cancer is recurrence of the disease due to the resistance of a small portion of the cancer cells to anticancer drugs. We have previously reported that human colon carcinoma cells that survived anticancer drugs were mainly CaSR-negative cells (11), and artificially knocking-down of CaSR in the cells reduced the cell sensitivity to anticancer drugs (11). This data suggested that CaSR could be exploited as a target for anticancer therapy, i.e., inducing CaSR in colon carcinoma cells may turn the drug-resistant cells to drug-sensitive cells. We found that calcitriol, the active form of vitamin D, enhanced 5-FU cytotoxic effect in the colon carcinoma cells through induction of CaSR (12), suggesting that manipulation of CaSR in human colon carcinoma cells could change the sensitivity of the cells to anticancer therapy. Calcitriol, however, is not applicable in clinic as an anticancer drug supplement due to its hypercalcemic side effect. Searching for other reagents that induce CaSR but have a better safety profile is, therefore, important for colon cancer therapy.

The mechanisms by which CaSR affects chemosensitivity are not fully understood. We examined survivin, TS, and E-cadherin to ß-catenin/TCF4 signaling system in the current study, since those proteins play pivotal roles in colon carcinoma cells in terms of cell malignancy, differentiation, apoptosis, and responsiveness to FOLFIRI, the current regimen for colon cancer. Survivin is an anti-apoptotic protein that blocks apoptotic process in carcinoma cells, whereas resistance to apoptosis is a key mechanism for the cells to resist anticancer drugs (29-31). TS is a key enzyme involved in the de novo synthesis of DNA and is the molecular target of 5-FU $(32,33)$. 5-FU poisons TS, disrupts DNA synthesis and kills cells. Cancer cells that express a high level of TS circumvent the efficacy of 5-FU and are relatively resistant to this drug $(34,35)$. Thus, induction of CaSR in the colon carcinoma cells suppressed survivin and TS expression, which decreased the resistance as well as increased sensitivity of the cells to 
anticancer treatment. Since CaSR not only suppressed protein expression of survivin and TS, but also inhibited gene promoter transcription of these proteins, we suggested that the suppression of the genes by CaSR mainly occurred at the level of transcriptional promotion and initiation. Activation of CaSR also stimulates the expression of E-cadherin $(8,9,36)$. E-cadherin is a major classical trans-membrane protein in epithelial cells. Upon activation, the cytoplasmic tail of Ecadherin interacts with B-catenin, preventing B-catenin translocation to nucleus where it forms a complex with TCF4 $(36,38)$. Formation and activation of $\beta$-catenin/TCF4 are prominent in driving the malignant progression of colon cancer $(36,37)$. Calcipotriol and lentinan suppressed the expression of survivin and TS through induction of CaSR in the human colon carcinoma cells. They also increased the localization of E-cadherin/ß-catenin complex in the cell membrane and suppressed TCF4 transcriptional activation activity in a CaSR-dependent manner. These results indicated that CaSR was the pivotal protein in generating the reactions in response to the treatment of the compounds.

In conclusion, calcipotriol and lentinan enhance sensitivity of human colon carcinoma cells to anticancer regimen FOLFIRI through their induction of CaSR expression, which leads to the suppression of survivin and TS gene expression, and the transcriptional activation of TCF4 in these cells. Since calcipotriol and lentinan have good safety profile in clinic, they have potential to be considered as supplements of colon cancer therapy.

\section{Acknowledgements}

We thank Dr Fengzhi Li (Dept. of Pharmacology and Therapeutics, Roswell Park Cancer Institute, Buffalo, NY) for providing survivin promoter luciferase reporter plasmid pLuc-2840, Dr Robert D. Ladner (Norris Comprehensive Cancer Center, University of Southern California, Los Angeles, $\mathrm{CA}$ ) for providing TS promoter luciferase reporter plasmid 3RG.

\section{References}

1. Brown EM, Pollak M and Hebert SC: The extracellular calciumsensing receptor: its role in health and disease. Annu Rev Med 49: 15-29, 1998

2. Rodland KD: The role of the calcium-sensing receptor in cancer. Cell Calcium 35: 291-295, 2004.

3. Hebert SC, Cheng S and Geibel J: Functions and roles of the extracellular $\mathrm{Ca}^{2+}$-sensing receptor in the gastrointestinal tract. Cell Calcium 35: 239-247, 2004

4. Larriba MJ and Munoz A: SNAIL vs vitamin D receptor expression in colon cancer: therapeutics implications. Br J Cancer 92: 985-989, 2005.

5. Kirchhoff P and Geibel JP: Role of calcium and other trace elements in the gastrointestinal physiology. World J Gastroenterol 12: 3229-3236, 2006.

6. Boman BM and Huang E: Human colon cancer stem cells: new paradigm in gastrointestinal oncology. J Clin Oncol 26: 2828-2838, 2008.

7. Chakrabarty S, Wang H, Canaff L, Hendy GN, Appelman H and Varani J: Calcium sensing receptor in human colon carcinoma interaction with $\mathrm{Ca}(2+)$ and 1,25-dihydroxyvitamin $\mathrm{D}(3)$. Cancer Res 65: 493-498, 2005.

8. Bhagavathula N, Hanosh AW, Nerusu KC, Appelman H, Chakrabarty $\mathrm{S}$ and Varani J: Regulation of E-cadherin and betacatenin by $\mathrm{Ca}^{2+}$ in colon carcinoma is dependent on calciumsensing receptor expression and function. Int J Cancer 121 $1455-1462,2007$
9. Chakrabarty S, Radjendirane V, Appelman H and Varani J: Extracellular calcium and calcium sensing receptor function in human colon carcinomas: promotion of E-cadherin expression and suppression of beta-catenin/TCF activation. Cancer Res 63. 67-71, 2003.

10. Bhagavathula N, Kelley EA, Reddy M, Nerusu KC, Leonard C, Fay K, Chakrabarty S and Varani J: Upregulation of calciumsensing receptor and mitogen-activated protein kinase signaling in the regulation of growth and differentiation in colon carcinoma. Br J Cancer 93: 1364-1371, 2005.

11. Liu G, Hu X, Varani J and Chakrabarty S: Calcium and calcium sensing receptor modulates the expression of thymidylate synthase, NAD(P)H:quinone oxidoreductase 1 and survivin in human colon carcinoma cells: promotion of cytotoxic response to mitomycin C and fluorouracil. Mol Carcinog 48: 202-211, 2009.

12. Liu G, Hu X and Chakrabarty S: Vitamin D mediates its action in human colon carcinoma cells in a calcium-sensing receptordependent manner: downregulates malignant cell behavior and the expression of thymidylate synthase and survivin and promotes cellular sensitivity to 5-FU. Int J Cancer 126: 631-639, 2010 .

13. Garrett JE, Capuano IV, Hammerland LG, Hung BC, Brown EM, Hebert SC, Nemeth EF and Fuller F: Molecular cloning and functional expression of human parathyroid calcium receptor cDNAs. J Biol Chem 270: 12919-12925, 1995.

14. Chikatsu N, Fukumoto S, Takeuchi Y, Suzawa M, Obara T, Matsumoto T and Fujita T: Cloning and characterization of two promoters for the human calcium-sensing receptor (CaSR) and changes of CaSR expression in parathyroid adenomas. $\mathrm{J}$ Biol Chem 272: 7553-7557, 2000.

15. Canaff L and Hendy GN: Human calcium-sensing receptor gene. Vitamin D response elements in promoters $\mathrm{P} 1$ and $\mathrm{P} 2$ confer transcriptional responsiveness to 1,25-dihydroxyvitamin D. J Biol Chem 277: 30337-30350, 2002.

16. Haussler MR, Haussler CA, Bartik L, Whitfield GK, Hsieh JC, Slater S and Jurutka PW: Vitamin D receptor: molecular signaling and actions of nutritional ligands in disease prevention. Nutr Rev 66: S98-S112, 2008

17. Fakih MG, Trump DL, Johnson CS, Tian L, Muindi J and Sunga AY: Chemotherapy is linked to severe vitamin D deficiency in patients with colorectal cancer. Int J Colorectal Dis 24: 219-224, 2009.

18. Cross HS, Lipkin M and Kállay E: Nutrients regulate the colonic vitamin D system in mice: relevance for human colon malignancy. J Nutr 136: 561-564, 2006.

19. Garland CF, Comstock GW, Garland FC, Helsing KJ, Shaw EK and Gorham ED: Serum 25-hydroxyvitamin D and colon cancer: eight-year prospective study. Lancet 2: 1176-1178, 1989.

20. Saraceno R andreassi L, Ayala F, Bongiorno MR, Giannetti A, Lisi P, Martini P, Peris K, Peserico A and Chimenti S: Efficacy, safety and quality of life of calcipotriol/betamethasone dipropionate (Dovobet) versus calcipotriol (Daivonex) in the treatment of psoriasis vulgaris: a randomized, multicentre, clinical trial. J Dermatolog Treat 18: 361-365, 2007.

21. Kidd PM: The use of mushroom glucans and proteoglycans in cancer treatment. Altern Med Rev 5: 4-27, 2000.

22. Zajickova K, Vrbikova J, Canaff L, Pawelek PD, Goltzman D and Hendy GN: Identification and functional characterization of a novel mutation in the calcium-sensing receptor gene in familial hypocalciuric hypercalcemia: modulation of clinical severity by vitamin D status. J Clin Endocrinol Metab 92: 2616-2623, 2007.

23. Ling X, Bernacki RJ, Brattain MG and Li F: Induction of survivin expression by taxol (paclitaxel) is an early event, which is independent of taxol-mediated G2/M arrest. J Biol Chem 279: 15196-15203, 2004.

24. Mandola MV, Stoehlmacher J, Muller-Weeks S, Cesarone G, Yu MC, Lenz HJ and Ladner RD: A novel single nucleotide polymorphism within the $5^{\prime}$ tandem repeat polymorphism of the thymidylate synthase gene abolishes USF-1 binding and alters transcriptional activity. Cancer Res 63: 2898-2904, 2003.

25. Albini A, Iwamoto Y, Kleinman HK, Martin GR, Aaronson SA, Kozlowski JM and McEwan RN: A rapid in vitro assay for quantitating the invasive potential of tumor cells. Cancer Res 47: 3239-3245, 1987.

26. Liu G, Bode A, Ma WY, Sang S, Ho CT and Dong Z: Two novel glycosides from the fruits of Morinda citrifolia (noni) inhibit AP-1 transactivation and cell transformation in the mouse epidermal JB6 cell line. Cancer Res 61: 5749-5756, 2001. 
27. Popat J, Matakidou A and Houlston RS: Thymidylate synthase expression and prognosis in colorectal cancer: a systemic review and meta-analysis. J Clin Oncol 22: 529-636, 2004

28. Notarbartolo M, Cervello M, Poma P, Dusonchet L, Meli M and Dalessandro N: Expression of the IAPs in multidrug resistant tumor cells. Oncol Rep 11: 133-136, 2004.

29. Altieri DC: Survivin in apoptosis control and cell cycle regulation in cancer. Prog Cell Cycle Res 5: 447-452, 2003.

30. Ferrario A, Rucker N, Wong S, Luna M and Gomer CJ: Survivin, a member of the inhibitor of apoptosis family, is induced by photodynamic therapy and is a target for improving treatment response. Cancer Res 67: 4989-4995, 2007.

31. Li F, Ambrosini G, Chu EY, Plescia J, Tognin S, Marchisio PC and Altieri DC: Control of apoptosis and mitotic spindle checkpoint by survivin. Nature 396: 580-584, 1998.

32. Marsh S: Thymidylate synthase pharmacogenetics. Invest New Drugs 23: 533-537, 2005.
33. Danenberg PV: Pharmacogenomics of thymidylate synthase in cancer treatment. Front Biosci 9: 2484-2494, 2004.

34. Chu E, Callender MA, Farrell MP and Schmitz JC: Thymidylate synthase inhibitors as anticancer agents: from bench to bedside. Cancer Chemother Pharmacol 52: S80-S89, 2003.

35. Rose MG, Farrell MP and Schmitz JC: Thymidylate synthase: a critical target for cancer chemotherapy. Clin Colorectal Cancer 1: 220-229, 2002.

36. Whitfield JF: Calcium, calcium-sensing receptor and colon cancer. Cancer Lett 275: 9-16, 2009.

37. MacLeod RJ, Hayes M and Pacheco I: Wnt5a secretion stimulated by the extracellular calcium-sensing receptor inhibits defective Wnt signaling in colon cancer cells. Am J Physiol Gastrointest Liver Physiol 293: G403-G411, 2007. 